\title{
Implication on our Universe of Pressure exerted by Multiverses in Multiple Dimensions
}

\author{
Saanchi Thawani \\ GGIS, Pune, India. \\ saanchithawani@gmail.com
}

\begin{abstract}
Recent experiments have provided scientific basis for the existence of multiverses - multiple physical universes located in space. String theory coupled with experiments with the quantum machine experiment further position multiverses not just as multiple universes in space, but also in time. While many papers have debated the scientific foundations for these concepts, few have evolved theories about the implications on earth of the existence of multiverses in space and time. In this paper, the author discusses the possible implications of multiverses due to pressure exerted by them in multiple dimensions. The author explores different effects of multiverses on physical phenomena experienced on earth such as earthquakes, volcanoes, and climate change, categorized by the levels of multiverse and the multiple dimensions in which pressure would be exerted.
\end{abstract}

\section{KEYWORDS}

Multiverse, Multiple Dimensions, String Theory, Inflation, Pressure, Space Time, Chaotic Inflation, Levels of Multiverse.

\section{ACADEMIC DISCIPLINE AND SUB-DISCIPLINES}

Physics- Astrophysics

\section{SUBJECT CLASSIFICATION}

Fundamental Astronomy and Astrophysics

\section{TYPE (METHOD/APPROACH)}

Qualitative

\section{CONTEXT}

Multiverses are alternate bubble like parallel/alternate universes, finite or infinite, existing in various dimensions and quantum states in the same vast space of which our universe is a part of. Several theories have proven the existence of multiverses, the most crucial being the Theory of inflation - Eternal Chaotic inflation ${ }^{[1][2]}$ and The String theory ${ }^{[3]}$.

Multiverses are present not only in space but also in time and multiple other undefined dimensions. String theory began developing in the 1970s which describes all known forces of nature at once with at least 10 physical dimensions thus laying down a foundation for the possibility of the existence of multiverses in not just in space and time but in multiple dimensions in addition to it.

In addition to existing in multiple dimensions, past discoveries have also confirmed that the universe is continuously expanding in both space and time. Astronomer Edwin Hubble's discovery ${ }^{[4]}$ in 1929 , Saul Perlmutter, Brian P. Schmidt and Adam G. Riess, 2011 Nobel prize laureates, in 1990 measured the rate of expansion of the universe ${ }^{[5]}$. More recently, the detection of gravitational waves by L.I.G.O. ${ }^{[6]}$ confirmed the presence of space time fabric ${ }^{[7]}$ and hence confirmed that the universe is continuously expanding in both space and time.

In this ever expanding multiverse, there will be some regions of space-time that have stopped expanding and some that are expanding at a rapid rate. The author believes that there will be some effects of this differential expansion of multiverses. In this paper, the author explores the effects of pressure of multiverses on each other in this ever expanding multiverse in multiple dimensions.

\section{SCOPE OF WORK}

In this paper, the author explores the effect of pressure in an expanding multiverse. The effect of pressure on different levels of multiverse, based on the dimensions of string theory and quantum properties is varied.

It must be noted that the existence of multiverses is not an independent theory. It is the implication and prediction of certain proven theories, and hence subject to debates and criticism in scientific forums. In this paper, the author assumes the reader is convinced about the existence of multiverses. The discussion of proofs for the existence of multiverses is outside the scope of this paper.

There also exist counter theories to the concept of expansion of the universe. In this paper, the author assumes that the theory of inflation of the universe holds true. The discussion of proofs for the validity of the concept of inflation is outside the scope of this paper.

This paper aims at discussing the presence of multiverse in all dimensions, including space time and its corresponding implications. 


\section{MULTIVERSES IN SPACE AND TIME 3.1 Multiverses}

Multiverses are the hypothetical ensemble of finite and infinite possible universes, including the universe in which we live. In order to appreciate the effect of pressure on multiverses, it is essential to first understand how multiverses are created.

13.8 billion years ago, our universe was compressed together in a dense and small mass. The bang, due to quantum fluctuations, had an inflating effect accompanied with the explosive scaling up of space time. Our universe began with a brief period of exponential expansion driven by a 'false vacuum ${ }^{[8]}$ which creates gravitational repulsion. The Theory of Chaotic Eternal Inflation ${ }^{[9]}$ states that in some regions of the universe, inflation stopped, but it still continues in remote parts. This decay of the false vacuum at different rates causes space time to bend in such a way that it gives rise to bubble or pocket universes.

At a subatomic particle level, based on quantum mechanics we know that phenomena conventionally forbidden by energy barriers are made possible by the process of tunneling ${ }^{[10]}$ through these energy barriers.

\subsection{Multiverses in space}

As discussed previously, it is inferred that multiverses lie in the pockets of space time created due to non-uniform inflation (Chaotic Eternal Inflation). Scientists have also predicted other areas in which multiverses could occur. Some of the predictions include the repeated regions of space time and layers of space time stacked over each other. Research by some scientists also posits that multiverses could be bubbles next to each other in the infinite space, or exist in various dimensions of space.

Multiverses exist beyond our observable horizon, needing us to rely on secondary evidence, such as imprints on radiation, to understand them better. Scientists observing the Cosmic Microwave Background at the U.S. Planck Data Center at Caltech noticed different polarizations and patterns in these imprints on radiation in 2015. On removing all known objects from the images obtained from the Planck satellites, scientists noticed that in some locations scattered patches and clumps were observed - these are termed, cosmic bruising ${ }^{[11]}$. These cosmic bruises are 4500 times brighter than what our current theories predict. There is a strong possibility that these clumps were caused by another universe bumping into our universe.

According to Einstein's theory of relativity, a negatively curved universe ${ }^{[12][13]}$ would act as strong evidence of the presence of multiverses. Recently discovered gravitational waves and Cosmic Microwave Background need to be studied more to confirm this.

One of the the biggest obstacles to studying multiverses is the fact that the bubbles constantly expand, sometimes even faster than the speed of light. As a result the information never is captured and we have to rely on data from collisions or cosmic bruising from radiation imprints.

\subsection{Multiverses in time and other dimensions}

Apart from the three fundamental dimensions- length, width, and depth (the $x, y$, and $z$ axis, respectively) scientists believe there are many more dimensions to matter. The String Theory is a theoretical framework in which the point particles of particle physics are replaced by one-dimensional objects called strings which propagate through space and interact with each other. This theoretical framework of Superstring Theory states that the universe exists in ten different dimensions.

The fourth dimension is time, which governs the properties of all known matter at any given point. The fifth and sixth dimensions are where the notion of possible worlds arises. In the sixth, would see a plane of possible worlds, where we could compare and position all the possible universes that start with same initial conditions. In the seventh dimension, we have access to the possible worlds that start with different initial conditions. The eighth dimension gives us a plane of such possible universe histories, which branches out infinitely (hence they are called infinities). In the ninth dimension, we can compare all the possible universe histories, starting with all the different possible laws of physics and initial conditions. In the tenth and final dimension, we arrive at the point in which everything possible and imaginable is covered. There are $10^{\wedge} 500$ different types of pocket universes in a String Theory Landscape ${ }^{[14]}$.

Let us now explore theories that support and posit phenomena that are relevant to this paper.

According to the M-theory ${ }^{[15]}$, the extended multi dimensional region, brane ${ }^{[16]}$, is an adequate hiding place for an entire universe. If branes collide, the results could be of high intensity - the Big Bang may have been created by such a collision. We could be living on a brane with infinite extra dimensions without noticing them. If this hypothesis is true, there is a large amount of space available for other universes.

With the existence of 4 dimensions, Albert Einstein showed that space and time are interchangeable and fundamentally the same entity. This is an effect which becomes much more noticeable at relativistic speeds approaching that of light. Because the universe always expands in space time, it is impractical to estimate the properties of the Universe at any given point of time.

According to quantum mechanics, the wave function allows a quantum particle to exist in several states at once, this phenomenon is called superposition ${ }^{[17]}$. When we take a measurement we only see one of these realities, but the others also exist. This is known as the 'many worlds interpretation' of quantum mechanics ${ }^{[18][19]}$. Each alternative quantum 


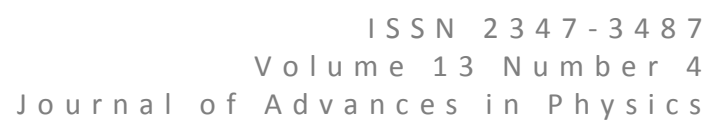

outcome must exist in a parallel reality, another world. To avoid wave function collapse, there must exist another universe and every quantum-transition may be splitting our universe into multiple copies.

To summarize the relevance of the theories that support the core idea in this paper - the string theory covers all possible dimensions $^{[20]}$ and laws of physics in the landscapes for the existence of multiverses. The theory of cosmic inflation provides the mechanism to generate these multiverses in the landscape. Quantum mechanics allows all possible transitions between all possible states.

\section{PRESSURE ON EARTH BY MULTIVERSES}

While many papers have debated the scientific foundations for the concepts of multi-verses and its existence in multiple dimensions, few have, few have evolved theories about the implications on earth of the existence of multiverses in space and time. In section 4.1, the author discusses the possible implications of multiverses due to pressure exerted by them in multiple dimensions. In section 4.2, the author explores different effects of multiverses on physical phenomena experienced on earth such as earthquakes, tsunamis and on the universe as a whole, categorized by the levels of multiverse and the multiple dimensions in which pressure would be exerted.

\subsection{Pressure in multiple dimensions}

Pressure is the continuous physical force exerted on or against an object by something in contact with it. Pressure is known to exist in multiple dimensions with different effects on the objects or the environment in contact with the source of the applied pressure.

Let us first explore the effects of pressure on multiple dimensions -

1 Dimension - Since it has only length, applied forces and pressure can only the change the length as contraction, expansion or bending

2 Dimensions- Either one, or both; length and width can be affected, which can also change the area.

3 Dimensions- Either length, width or height or different combinations of the same. Can affect the volume as a whole.

4 Dimensions- Since space(the 3dimensions) and time are interwoven, it can change curvature of spacetime. Time can be varied- slowed, made faster, altered..

5-6-7 Dimensions - As they Propose the existence of new possible worlds of different timelines, it can change the number of possibilities of new worlds and alter the time lines.

8-9 Dimensions- proposes infinities and has hence can have infinite variations

10 Dimensions- No limits.

Just like the effects of pressure can be interpreted different in different dimensions, the multiverse, based on its dimensions, effects, physical laws and its multiple interpretations has been categorized into various levels ${ }^{[21]}$, these 4 being the most widely accepted variants.

\section{Level 1}

Space is infinite and will contain an infinite number of Hubble volumes ${ }^{[22]}$, all having the same physical laws and physical constants. Estimations say that identical volume to ours should be about $10^{10115}$ meters away from us.

\section{Level 2}

As suggested by Chaotic inflation and String theory landscape, infinite pocket universes and the multiverse as a whole is expanding and will continue doing so forever, but some regions of space stop stretching, expanding and form distinct bubbles : the laws of physics are different in each of these pocket universes. Such bubbles are like the early stages of level I multiverses. Different bubbles may experience different breaking, which results in different properties, such as different physical constants.

Level 3

This is the 'many world' interpretation of quantum mechanics as discussed previously in the paper.. There is a range of possible observations, each with a different probability. In Level III the multiverse exists on quantum branches in infinitedimensional Hilbert space ${ }^{[23]}$.

\section{Level 4}

Level 4 consists of totally disconnected realities governed by different mathematical equations.. Worlds corresponding to different sets of initial conditions, physical constants, or altogether different equations are the norm in such a multiverse.

To illustrate this - in Level 1, there could just be pressure applied on Earth causing the Earth to bulge. but because of Level 3, time could be getting compressed and sometimes things appear to happen faster than normal. 


\subsection{Implications on earth of pressure exerted by multiverses}

The effect of pressure in general has been discussed, this part of the paper focuses on the effects more specific towards the multiverse, our universe and the earth and the 4 basic dimensions of space time.

\section{Table 1. Effect of pressure in different levels of Multiverse}

\begin{tabular}{|c|c|c|c|c|}
\hline & Level 1 Multiverse & Level 2 Multiverse & Level 3 Multiverse & $\begin{array}{c}\text { Level } 4 \\
\text { Multiverse }\end{array}$ \\
\hline $\begin{array}{l}\text { Characteristics of } \\
\text { this level of } \\
\text { multiverse }\end{array}$ & $\begin{array}{c}\text { Temperature } \\
\text { constant laws apply. } \\
\text { All have same } \\
\text { physical laws. }\end{array}$ & $\begin{array}{c}\text { Expansion laws do } \\
\text { not apply as level } 2 \\
\text { multiverses } \\
\text { experience partial and } \\
\text { differential expansion. }\end{array}$ & $\begin{array}{l}\text { Multiverses at this } \\
\text { level are possible } \\
\text { alternates to the real } \\
\text { universe that we live } \\
\text { in. }\end{array}$ & $\begin{array}{l}\text { Multiverses at level } 4 \text { are } \\
\text { described purely by } \\
\text { mathematical equations } \\
\text { and numbers. }\end{array}$ \\
\hline Space & $\begin{array}{c}\text { Represented by the } 3 \\
\text { dimensions - length, } \\
\text { width, height }\end{array}$ & $\begin{array}{l}\text { Represented by the } 3 \\
\text { dimensions - length, } \\
\text { width, height }\end{array}$ & $\begin{array}{c}\text { Represented by a } \\
\text { wave function in } 3 \\
\text { dimensions - length, } \\
\text { width, height }\end{array}$ & $\begin{array}{l}\text { Represented by the } 3 \\
\text { dimensions - length, } \\
\text { width, height }\end{array}$ \\
\hline Time & $\begin{array}{l}\text { No existence of time } \\
\text { in level } 1 \text { multiverses }\end{array}$ & $\begin{array}{l}\text { May or may not affect } \\
\text { time. Level } 2 \\
\text { multiverses have } 3+1 \\
\text { dimensions }\end{array}$ & $\begin{array}{c}\text { Time evolution exists } \\
\text { if the wave function } \\
\text { is unitary }\end{array}$ & $\begin{array}{l}\text { Affects time and can } \\
\text { cause its compression or } \\
\text { dilation }\end{array}$ \\
\hline $\begin{array}{l}\text { Other dimensions } \\
\text { beyond space and } \\
\text { time }\end{array}$ & $\begin{array}{l}\text { No, only } 3 \\
\text { dimensions }\end{array}$ & $\begin{array}{c}\text { Yes, may exist in } \\
\text { various dimensions } \\
\text { beyond space and } \\
\text { time }\end{array}$ & $\begin{array}{c}\text { May exist in } \\
\text { infinitely- } \\
\text { dimensional Hilbert } \\
\text { Space }\end{array}$ & $\begin{array}{l}\text { Different dimensions exist } \\
\text { as predicted by different } \\
\text { mathematical equations. } \\
\text { These dimensions may } \\
\text { not reside in the same } \\
\text { space time though }\end{array}$ \\
\hline $\begin{array}{l}\text { Potential } \\
\text { phenomena due to } \\
\text { pressure on our } \\
\text { universe by } \\
\text { multiverses }\end{array}$ & $\begin{array}{l}\text { Bulging of earth at } \\
\text { the equator, } \\
\text { expansion of our } \\
\text { universe, tectonic } \\
\text { shifts, earthquakes, } \\
\text { tidal movement }\end{array}$ & $\begin{array}{l}\text { Volcanoes, Non- } \\
\text { uniform climate } \\
\text { changes across the } \\
\text { globe, Bermuda } \\
\text { triangle, presence of } \\
\text { gravitational waves }\end{array}$ & $\begin{array}{l}\text { Collapse of the } \\
\text { universe, creation of } \\
\text { another big bang, } \\
\text { Clairvoyant / psychic } \\
\text { behavior of } \\
\text { individuals }\end{array}$ & $\begin{array}{l}\text { Random appearances by } \\
\text { aliens - UFO sightings }\end{array}$ \\
\hline
\end{tabular}

Let us now explore the different effects of multiverses on physical phenomena experienced on our universe, and hence our planet earth, such as earthquakes, volcanoes, and climate change, categorized by the levels of multiverse and the multiple dimensions in which pressure would be exerted.

\section{Effect of pressure on Level 1 multiverses}

Pressure from multiverses acting at different points of our universe could explain the expansion of our universe.

Earthquakes and tsunamis are caused by movements of tectonic plates which could in turn be caused due friction between a neighbouring multiverse and our universe.

\section{Effect of pressure on Level 2 multiverses}

The gravitational waves detected by LIGO could be from the application of pressure by a bubble universe onto us.

The transition of the earth's climate from a freezing ice age to the current state of global warming could be explained by the increase in separation of earth from a deep cold alternate universe due to the expansion of ours.

\section{Effect of pressure on Level 3 multiverses}

We know that our own universe is expanding at a rate of about 3.2 kilometers per second per megaparsec ${ }^{[24]}$. Multiverses surrounding us, may expand just as ours, but not necessarily at equal rates, or even uniformity. There is a possibility that their rate of expansion could exert tremendous pressure along space time boundaries, such that it exceeds our universe's expansion rate and overpowers the cosmological constant. This will result in our universe getting compressed into highly dense, intensive, pressurised, hypertensive mass, that cannot withstand any more pressure - thus resulting in the collapse of our universe. 
Conversely, pressure from our our universe may result in mass with life in a tiny space getting heated and reactivated. This may potentially regenerate life as we know it in another universe.

\section{Effect of pressure on Level 4 multiverses}

Level 4 multiverses pose endless probabilities, and variations. Since the multiple universes are disconnected and varied by various mathematical equations, their effect cannot be accurately predicted given our mental model of science today.

\section{CONCLUSIONS AND FUTURE WORK}

Although the theory of higher dimensional space has not been verified, almost 5,000 papers, have been published in physics literature concerning higher dimensional theories. Some observations such as polarizations of CMB are taking us a step closer towards the multiverse. Some basic phenomena we experience, such as earthquakes, tsunamis, climate change expose us to the possibilities of the effects of pressure on multiverse which has been discussed in this paper.

In this paper the author has covered the effects of pressure by primarily focussing only on the basic 4 dimensions. The exploration of possibilities of the effect of pressure beyond 4 dimensions, as briefly discussed in section 4 , open up a whole new realm of science.

The author hopes that this paper lays the foundation for exploration of the effects of multiple phenomena such as temperature, humidity beyond pressure on the multiverse with mathematical basis that was not covered in this paper. More research on the multiple dimensions of string theory, improvised and refined analysis of the CMB will help uncovering the impact of multiple dimensions. Discovering the mysteries of dark matter ${ }^{[25]}$, black holes ${ }^{[26]}$, worm-holes ${ }^{[27]}$, and other similar phenomena with the lens of multiverse in multiple dimensions would help uncover new properties in physics.

\section{REFERENCES}

Austin, A. (1996) Annotated List of World Wide Web Technical Writing and Computer-Aided Composition Resources [Online]. Available: http://wwwcsif.cs.ucdavis.edu/ austina/cai.html.

1. Alan H. Guth (1999) Eternal Inflation. [Online] http://cds.cern.ch/record/485381/files/0101507.pdf

2. Vilenkin Alexander (2011) The Principle of Mediocrity. [Online] http://cosmos2.phy.tufts.edu/Vilenkin.Whitrow.pdf

3. Wikipedia (2017) String Theory. [Online] https://en.wikipedia.org/wiki/String_theory

4. Kragh, H. \& Smith, R. W. (2003) Who discovered the expanding universe? [Online] http://adsabs.harvard.edu/full/2003HisSc..41..141K

5. The Royal Swedish Academy of Sciences (2011) The 2011 Nobel Prize in Physics - Press Release. [Online] https://www.nobelprize.org/nobel_prizes/physics/laureates/2011/press.html

6. LIGO Caltech (2016) Gravitational Waves Detected 100 Years After Einstein's Prediction- Press release. [Online] https://www.ligo.caltech.edu/news/ligo20160211

7. Luke Mastin (2009) Space Time [Online] http://www.physicsoftheuniverse.com/topics_relativity_spacetime.html

8. Wikipedia (2017) False Vacuum. [Online] https://en.wikipedia.org/wiki/False_vacuum

9] Andrei Linde (1994) The Self-Reproducing Inflationary Universe [Online] http://web.stanford.edu/ alinde/1032226.pdf

10. http://abyss.uoregon.edu/ js/glossary/quantum_tunneling.html

11. MIT Tech Review (2010) Astronomers Find First Evidence of Other Universes. [Online]

https://www.technologyreview.com/s/421999/astronomers-find-first-evidence-of-other-universes/

12. Yasunori Nomura (2015) Is this the only universe. [Online] http://physics.berkeley.edu/news-events/news/20150728/isthis-the-only-universe-yasunori-nomura-quoted

13. Ron Cowen (2013) Universe May Be Curved, Not Flat. [Online] https://www.scientificamerican.com/article/universemay-be-curved-not-flat/

14. Susskind (2003) The Anthropic Landscape of String Theory. [Online] https://arxiv.org/pdf/hep-th/0302219.pdf

15. Michio Kaku (2017) M-Theory: The Mother of all SuperStrings [Online]http://mkaku.org/home/articles/m-theory-themother-of-all-superstrings/

16. Patricia Schwarz () Is there a more fundamental theory? [Online] http://www.superstringtheory.com/basics/basic7.html

17. Luke Mastin (2009) Superposition, Interference, and Decoherence. [Online] http://www.physicsoftheuniverse.com/topics_quantum_superposition.html

18. Lev Vaidman (2014) Many-Worlds Interpretation of Quantum Mechanics [Online]

https://plato.stanford.edu/entries/qm-manyworlds/ 
19. Sean Carroll (2014) Why the Many-Worlds Formulation of Quantum Mechanics Is Probably Correct [Online] http://www.preposterousuniverse.com/blog/2014/06/30/why-the-many-worlds-formulation-of-quantum-mechanics-isprobably-correct/

20. Matt Williams (2014) A universe of 10 dimensions [Online] https://phys.org/news/2014-12-universe-dimensions.html

21. Max Tegmark (2003) WELCOME TO MY CRAZY UNIVERSE

[Online] http://space.mit.edu/home/tegmark/crazy.html

22. Wikipedia (2016) Hubble Volume [Online] https://en.wikipedia.org/wiki/Hubble_volume

23. University Of California, Davis [US] Hilbert Space [Online] https://www.math.ucdavis.edu/ hunter/book/ch6.pdf

24. Mike Wall (2016) Universe is expanding faster than thought. [Online] http://www.space.com/33061-universeexpanding-faster-than-thought-hubble.html

25. Gianfranco Bertone, Dan Hooper and Joseph Silk (2008) Particle Dark Matter: Evidence, Candidates and Constraints [Online] https://arxiv.org/pdf/hep-ph/0404175.pdf

26. Ligo Scientific Collaboration (2015-16) Observation of Gravitational Waves from a Binary Black Hole Merger [Online] http://www.ligo.org/science/Publication-GW150914/

27. Andrew Friedman. Wormholes: Space Machines and Time Machines [Online] http://web.mit.edu/asf/www/Wilfred_Wormhole/wormhole.pdf

\section{Author' biography with Photo}

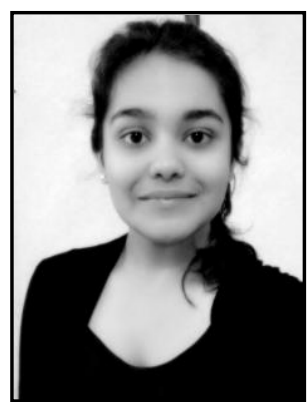

Saanchi Thawani has research interests in deep space exploration and cosmology. She has made 21 preliminary discoveries of moving objects under an asteroid search campaign and has won two Digital Badges from NASA. Saanchi pursues cutting edge projects with DoNew and studies at the GGIS in Pune, India. 